\title{
Article
}

\section{Ionothermal Synthesis of Triclinic SAPO-34 Zeolites}

\author{
Li Han ${ }^{1,2}, *$, Xinxin Yan ${ }^{2}$, Lulu Guo ${ }^{2}$, Yanan Duan ${ }^{2}$, Zheng Wang ${ }^{3}$, Tianliang $\mathrm{Lu}^{2}$, Jun $\mathrm{Xu}^{2}$, Yuzhong Zhan ${ }^{2}$ \\ and Jianfeng Wang ${ }^{1, *}$
}

1 School of Ecology and Environment, Zhengzhou University, Zhengzhou 450001, China

2 School of Chemical Engineering, Zhengzhou University, Zhengzhou 450001, China; xinxinyan0131@163.com (X.Y.); gllzzu@163.com (L.G.); DuanYN_ZZU@163.com (Y.D.); lutianliang@zzu.edu.cn (T.L.); jxuzzu@sohu.com (J.X.); zhanyz@zzu.edu.cn (Y.Z.)

3 State Key Laboratory of High-Efficiency Utilization of Coal and Green Chemical Engineering, Institute of Chemistry and Chemical Engineering, Ningxia University, Yinchuan 750021, China; wzheng@nxu.edu.cn

* Correspondence: lihan@zzu.edu.cn (L.H.); wangjf@zzu.edu.cn (J.W.)

Citation: Han, L.; Yan, X.; Guo, L.; Duan, Y.; Wang, Z.; Lu, T.; Xu, J.;

Zhan, Y.; Wang, J. Ionothermal Synthesis of Triclinic SAPO-34 Zeolites. Catalysts 2021, 11, 616. https://doi.org/10.3390/ catal11050616

Academic Editor: Wladimir Reschetilowski

Received: 11 April 2021

Accepted: 4 May 2021

Published: 11 May 2021

Publisher's Note: MDPI stays neutral with regard to jurisdictional claims in published maps and institutional affiliations.

Copyright: (C) 2021 by the authors. Licensee MDPI, Basel, Switzerland. This article is an open access article distributed under the terms and conditions of the Creative Commons Attribution (CC BY) license (https:// creativecommons.org/licenses/by/ $4.0 /)$.

\begin{abstract}
A triclinic SAPO-34 molecular sieve was synthesized ionothermally. The as-synthesized products were characterized by powder X-ray diffraction (XRD), scanning electron microscope (SEM), nuclear magnetic resonance (NMR), fourier infrared spectrometer (FT-IR) and thermogravimetric (TG) analyses. The formation mechanism of the hierarchical porous triclinic SAPO-34 zeolites and the factors affecting the morphology of the SAPO-34 molecular sieve were investigated. The results show that the formation mechanism of the hierarchical pores is in accordance with Ostwald ripening theory, and the accumulation of grains constitutes the existence of mesopores and macropores. The crystallization temperature, ionic liquid type, and organic amines can effectively change the morphology and crystallinity of the SAPO-34 molecular sieve. The crystallization temperature, ionic liquid and template have great influence on the (111) crystal plane, thus affecting the morphology of the molecular sieve. Moreover, it can be proven through NMR and TG analyses that ionic liquids and organic amines can be used as structure directing agents together.
\end{abstract}

Keywords: ionic liquid; ionothermal synthesis; SAPO-34; molecular sieve

\section{Introduction}

Zeolites and zeotypes have emerged as some of the most significant crystalline microporous materials. Owing to their structural variability and widespread industrial applications, they have been extensively studied [1-3]. The silicoalumino-phosphate zeolite SAPO-34 with a CHA framework is an important microporous crystal with uniform and intricate channels, high specific surface area, adsorption capacity, and high thermal and hydrothermal stabilities, which have been widely used in catalysis [4,5], adsorption, ion-exchange, separation [6], etc. The performance in these fields is greatly influenced by the structure and properties of the SAPO-34 zeolite. The number and utilization of active sites, the ratio of weak acid to strong acid sites and the specific surface area can be changed by changing the morphology and size of zeolites [7-9]. The introduction of mesopores or macropores into microporous molecular sieves can greatly improve the mass transfer rate, especially in catalytic reactions, contributing to the diffusion of larger molecules, reduce the rate of formation of coke and prolong the catalytic lifetime [4]. Moreover, the synthesis methods and conditions have a substantial impact on the structure and properties of SAPO-34 zeolite.

Conventionally, SAPO-34 zeolite is mainly synthesized by a hydrothermal method, which produces high autogenous pressure and a long crystallization time. As the solvent and structure directing agent (SDA) are two different species, there is always competition between the solvent and SDA to interact with the growing framework [10]. Moreover, synthesis under high pressure must use an autoclave lined with Teflon, which limits 
production to batch processes. Therefore, it is necessary to explore a new preparation method to replace the traditional hydrothermal method.

In 2004, Morris et al. reported a new synthesis method of zeolite, i.e., ionothermal synthesis [11]. This method uses an ionic liquid as the solvent and SDA, which eliminates the competition between the solvent-framework and the template-framework, and avoids the obstruction of the structure-directing effect of the solvent [11-13]. Ionic liquids can be defined as liquids composed entirely of ions [14]. They have an extremely low vapor pressure even at high temperature, high solubility for zeolite precursors, tunability of physicochemical properties and organization in their liquid structure, providing a convenient and innovative space for the synthesis of molecular sieves [15-17]. In addition, the ionic liquid can be efficiently recovered and reused. Therefore, the ionothermal synthesis method can provide a shorter production cycle.

To date, most research has focused on the synthesis of aluminophosphates (AlPOs) using ionothermal synthesis. Parnham et al. [12] investigated aluminophosphate zeolite materials in sealed autoclaves with alkyl chain lengths changing from 2 to 5 carbons in 1-alkyl3-methyl imidazolium bromide ionic liquids. Bengueddach et al. [18] prepared AlPO-34, chabazite-like (CHA) zeolite by using 1-ethyl-3-methyl imidazolium chloride ([EMIm]Cl) as the solvent. Tian et al. [19] synthesized a CHA-type aluminophosphate molecular sieve membrane in 1-ethyl-3-methyl imidazolium bromide ([EMIm]Br). Lee et al. [20] prepared microporous triclinic $\mathrm{AlPO}_{4}-34$ by using 1,2,3-trimethylimidazolium (123-TMI). There are few reports on the synthesis of SAPOs using ionic liquids. Lin et al. [21] prepared silicoaluminophosphate zeotype with an LTA structure (SAPO-LTA) using an ionothermal approach in a commercial 1-ethyl-3-methyl imidazolium ([EMIm]+) ionic liquid. Zhao et al. [22] synthesized a hierarchically structured SAPO- 5 molecular sieve by a microwave-assisted ionothermal method in a eutectic mixture solvent. To the best of our knowledge, there are only two reports on SAPO-34 molecular sieves. Sastre et al. [23] reported that the direct addition of $\mathrm{Si}$ sources to an aluminophosphate and ionic liquid (1-ethyl-3-methyl imidazolium) mixture cannot synthesize the CHA-structured materials, and the addition of further $\mathrm{V}$ ions is able to direct CHA materials. Stark et al. [24] mentioned the synthesis of SAPO-34 by 1-methylimidazole addition in ionic liquids. Thus, there is not enough literature to fully understand the ionothermal synthesis of SAPO-34 zeolite. Therefore, this work conducted a detailed and systematic study on the synthesis of SAPO-34 zeolite to determine the effects of reaction temperature, ionic liquid type, and organic amines on the structure and morphology of the resulting samples.

\section{Results and Discussion}

\subsection{Effect of Crystallization Temperature}

The powder X-ray diffraction (XRD) patterns are shown in Figure 1. The characteristic peaks at $9.68^{\circ}, 15.97^{\circ}, 20.2^{\circ}, 26.0^{\circ}$, and $31.0^{\circ}$ confirm the structure of the triclinic SAPO-34 molecular sieve in these materials. The intensity of the CHA reflection peaks increased greatly with increasing temperature from $150{ }^{\circ} \mathrm{C}$ to $180^{\circ} \mathrm{C}$. The characteristic peaks of the AEI structure appeared at approximately $24.5^{\circ}$ and $25.4^{\circ}$ when the temperature rose to $200{ }^{\circ} \mathrm{C}$.

Figure 2 shows scanning electron microscope (SEM) images of the samples, and the morphology of the triclinic SAPO-34 molecular sieve changed greatly with increasing temperature. Sample $\mathrm{S} 1\left(150^{\circ} \mathrm{C}\right)$ shows spherical agglomerates composed of many micrometer-sized flaky crystals, while sample $\mathrm{S} 1\left(180^{\circ} \mathrm{C}\right)$ changes to a spherical aggregate composed of many cubic crystals, and each cubic crystal is approximately $1 \mu \mathrm{m}$. Increasing the reaction temperature from $150{ }^{\circ} \mathrm{C}$ to $180^{\circ} \mathrm{C}$ promotes the growth of the (100) and (111) crystal planes. Sample $\mathrm{S} 1\left(200^{\circ} \mathrm{C}\right)$ is a spherical aggregate composed of cubic crystals, but there are a few rhombus cubes. The growth of the (111) crystal plane is further promoted with increasing crystallization temperature, which favors the formation of cubic crystals. 


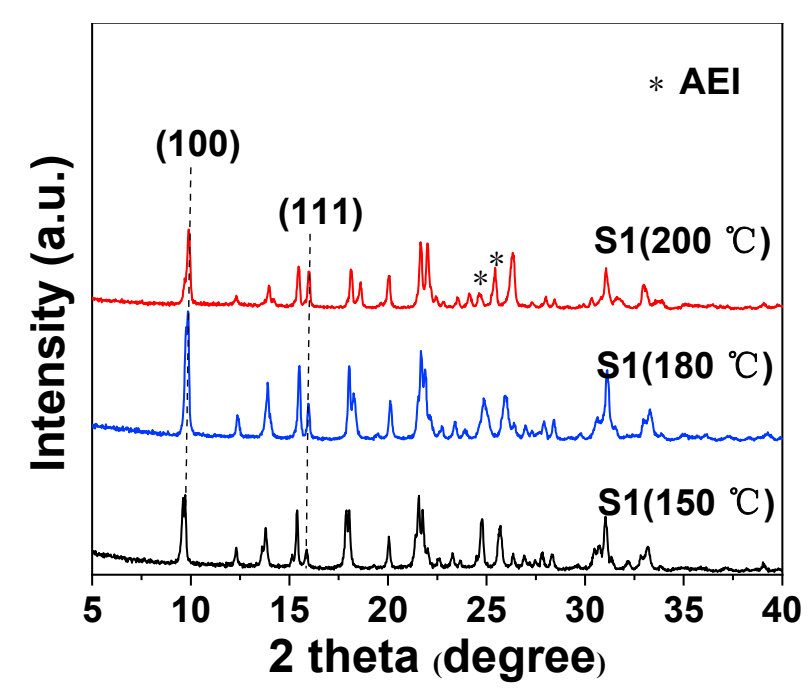

Figure 1. Powder X-ray diffraction (XRD) patterns of the samples synthesized at different crystallization temperatures.

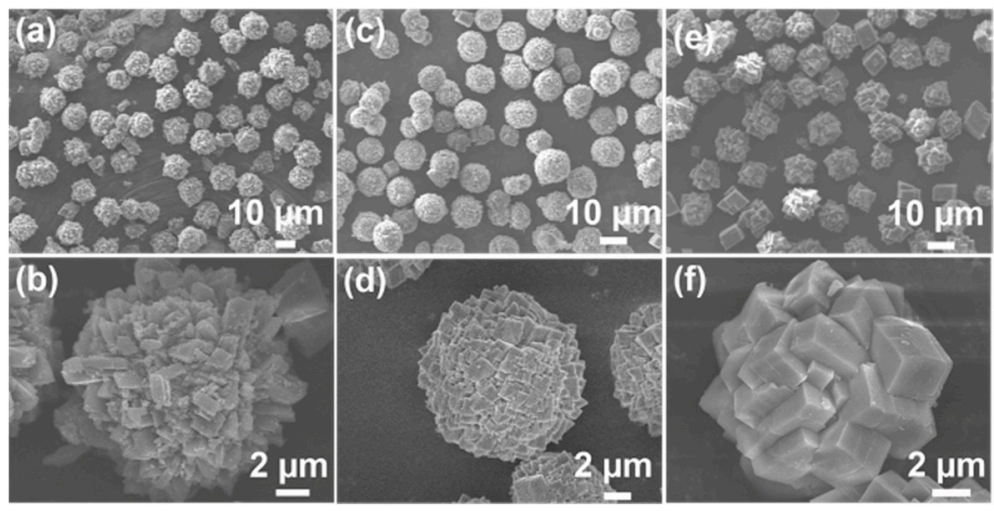

Figure 2. Scanning electron microscope (SEM) images of the samples synthesized at different crystallization temperatures $\left((\mathbf{a}, \mathbf{b})\right.$ for S1 $\left(150^{\circ} \mathrm{C}\right),(\mathbf{c}, \mathbf{d})$ for S1 $\left(180^{\circ} \mathrm{C}\right)$, and $(\mathbf{e}, \mathbf{f})$ for $\mathrm{S} 1\left(200^{\circ} \mathrm{C}\right)$ ).

The above results show that crystallization temperature is an important factor influencing the SAPO-34 molecular sieve. In the crystallization process of the triclinic SAPO-34 molecular sieve, a lower crystallization temperature is beneficial for the formation of flaky crystals, while a higher crystallization temperature is beneficial for the growth of the (111) crystal plane and the generation of large cubic crystals.

\subsection{Synthesis of SAPO-34 in [EMIm]Cl}

Cation [EMIm] ${ }^{+}$with smaller size is easier to form molecular sieves with an eightmembered ring $\mathrm{CHA}$ structure; therefore, [EMIm] $\mathrm{Cl}$ ionic liquid is firstly used to synthesize SAPO-34 molecular sieves.

A triclinic SAPO-34 molecular sieve was synthesized in [EMIm]Cl. The silicon contents and HF have a great influence on the synthesis, structure and morphology of the SAPO-34 molecular sieves.

Figure S1 shows the XRD patterns of samples $\mathrm{AlPO}_{4}-34, \mathrm{~S} 1-\mathrm{Si}-0.1, \mathrm{~S} 1-\mathrm{Si}-0.3$ and S1Si-0.5. The SAPO-34 molecular sieve was prepared with a low content of silicon by using ionothermal synthesis. The detailed XRD data are shown in Table S1, and the decrease in interplanar spacing and the shift in the diffraction peak prove that silicon atoms enter the framework of the SAPO-34 molecular sieve. With increasing silicon molar amount, the morphology of the SAPO-34 molecular sieve has no obvious change, and only the overall sizes of cubic crystals and spherical aggregates decrease (Figure S2). As shown in Figure 3, 
samples S1-Si-0.1, S1-Si-0.3 and S1-Si-0.5 have absorption peaks at $1215 \mathrm{~cm}^{-1}, 1100 \mathrm{~cm}^{-1}$, $735 \mathrm{~cm}^{-1}, 645 \mathrm{~cm}^{-1}, 560 \mathrm{~cm}^{-1}, 525 \mathrm{~cm}^{-1}$ and $485 \mathrm{~cm}^{-1}$, which prove the synthesis of SAPO-34 zeolites, and the peak at $480 \mathrm{~cm}^{-1}$ is the T-O bending vibration peak of $\mathrm{SiO}_{4}$ [25], which confirms that silicon enters the skeleton of the crystal.

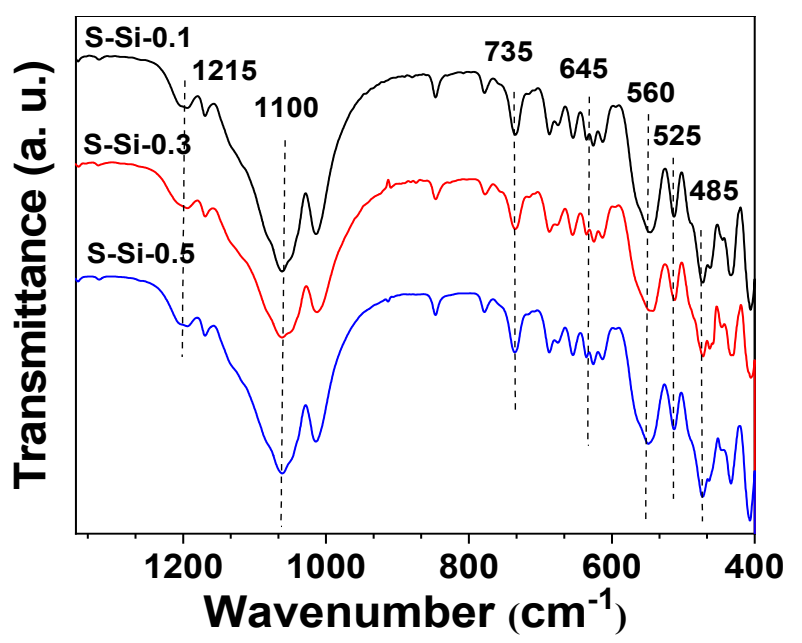

Figure 3. Fourier infrared spectrometer (FT-IR) spectra of SAPO-34 molecular sieves synthesized in different molar amounts of Si.

Figure 4 depicts resonances at -107 and $-111.8 \mathrm{ppm}$, which correspond to $\mathrm{Si}(\mathrm{OAl})(\mathrm{OSi})_{3}$ and $\mathrm{Si}(\mathrm{OSi})_{4}$, respectively $[24,26]$, indicating that the substitution of Si follows the SM2 and SM3 mechanisms. The combination of SM2 and SM3 mechanisms leads to the formation of silica-rich domains that are commonly known as Si-islands. The EDS results (Table S2) point out that the $\mathrm{Si} / \mathrm{Al}$ ratio is increased by increasing the amount of $\mathrm{Si}$ in the synthesized gel, which also proves the formation of Si-islands.

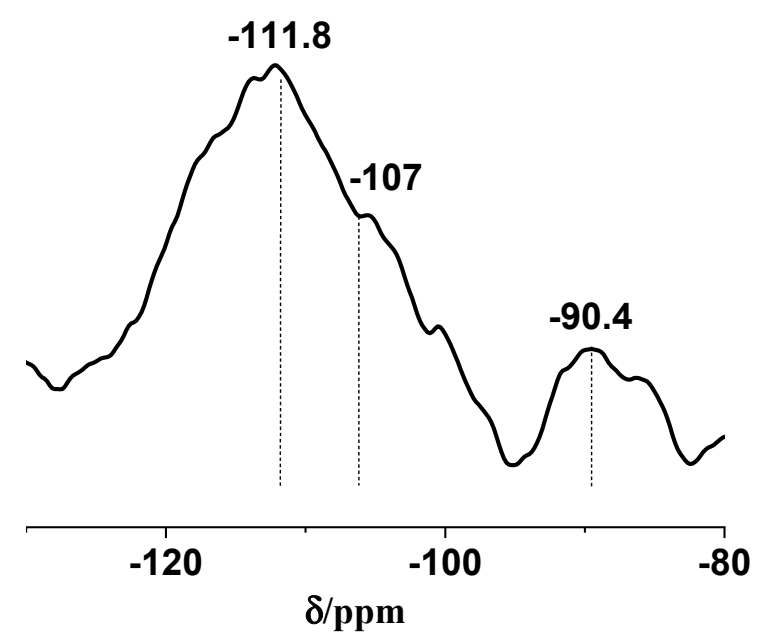

Figure 4. ${ }^{29}$ Si nuclear magnetic resonance (NMR) spectra of sample S-Si-0.3.

The influence of HF was investigated by performing the synthesis in solution with different amounts of HF. XRD patterns of samples under different amounts of HF are shown in Figure S3. SAPO-34 molecular sieves cannot be prepared without the addition of HF, and S1-F-0 has an amorphous morphology. A proper amount of HF is beneficial for the reaction of phosphorus and aluminum sources with $\mathrm{F}^{-}$, which can generate more crystal nuclei and promote the formation of the molecular sieve, while excessive HF can inhibit the release rate of phosphorus and aluminum species in the system, reduce the $\mathrm{pH}$ value of the system, and is not conducive to crystal growth. A SAPO-34 molecular sieve 
with uniform morphology and high crystallinity was obtained when the amount of HF was 0.7 (Figure S4).

Figure S5a shows the $\mathrm{N}_{2}$ adsorption/desorption isotherms of the SAPO-34 samples with different silicon contents. The steep rise followed by flat curves at low partial pressure results from the filling of the micropores, while hysteresis loops were observed which are ascribed to the presence of mesopores. The uptakes near the saturation pressure in the isotherms indicate that macropores exist in the samples. The detailed data are shown in Table S2. The mesoporous size distribution of the sample is $3 \sim 30 \mathrm{~nm}$, as shown in Figure S5b. The total surface areas of all samples are higher than $512 \mathrm{~m}^{2} / \mathrm{g}$. With the increase in the silicon source in the synthesis system, the specific surface area and mesoporous volume of the molecular sieve increase, and sample S-Si- 0.5 has the largest external surface area $\left(57 \mathrm{~m}^{2} / \mathrm{g}\right)$ and mesoporous volume $\left(0.17 \mathrm{~cm}^{2} / \mathrm{g}\right)$.

To study the formation process of hierarchical porous structures, comparative timedependent experiments were conducted. Figures S6 and S7 show the XRD patterns and SEM images. The results show that the complete SAPO-34 characteristic diffraction peak and porous structure appeared at $3 \mathrm{~h}$. By extending the reaction time further, the relative crystallinity increased, and after $48 \mathrm{~h}$, it resembled the maximum relative crystallinity. The triclinic SAPO-34 molecular sieve started to nucleate, and metastable small grains appeared slowly in the system after crystallization treatment for $3 \mathrm{~h}$. After prolonging the crystallization time to $6 \mathrm{~h}$, the triclinic SAPO- 34 molecular sieve showed a spherical aggregate composed of 100 200 nm small grains and the crystal surface was not completely covered by small grains. While the crystal grows rapidly with the extension of crystallization time to $12 \mathrm{~h}$, the overall size of the crystal gradually increases from $4 \mu \mathrm{m}$ to $10 \mu \mathrm{m}$. The crystal grows completely after $48 \mathrm{~h}$, and the aluminosilicate ions in the liquid phase cannot satisfy the further growth of the crystal, so the overall size of the crystal will not increase significantly.

The proposed process of forming triclinic SAPO- 34 zeolite with a hierarchically porous structure is shown in Scheme 1. The formation mechanism was in accordance with the Ostwald ripening theory. During the synthesis of the molecular sieve, the smaller grains will gradually dissolve into the surrounding medium due to the larger curvature and higher energy content and then precipitate on the surface of the larger grains [12], forming spherical agglomerates composed of small grains. With the extension of crystallization time, the crystals continue to grow and finally form a hierarchical porous triclinic SAPO-34 molecular sieve with more thermodynamically stable cubic crystals. The accumulation of grains constitutes the existence of mesopores and macropores.

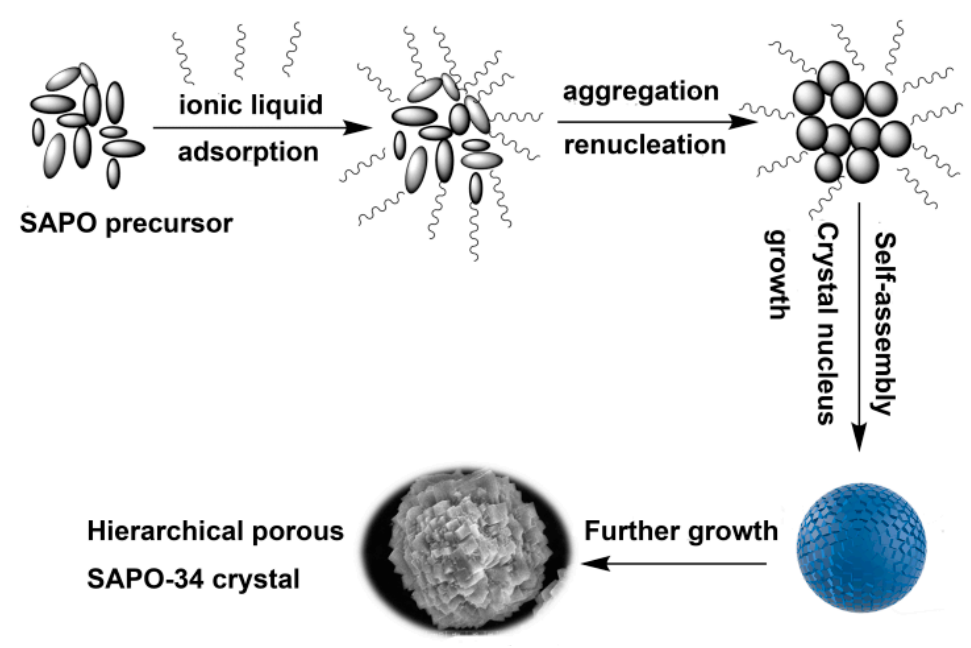

Scheme 1. Schematic drawing of the formation mechanism of hierarchical porous triclinic SAPO-34 molecular sieves in ionic liquids. 


\subsection{Effect of Types of Ionic Liquid}

To study the influence of cations on the triclinic SAPO-34 molecular sieve, ionic liquids with the same anions but different cations were used. The structure of the used ionic liquids is shown in Figure S8. The SAPO-34 molecular sieves synthesized in either [EMIm]Cl (S-EM-C) or [EMIm]Br (S-EM-B) were compared to those obtained in either [BMIm]Cl (S-BM-C) or [BMIm] Br (S-BM-B). The morphology of the sample changed from spherical to cubic as the cation of the ionic liquid was changed from 1-ethyl-3-methyl imidazolium to 1-butyl-3-methyl imidazolium, while the size of the sample decreased. Cations in short carbon chain substituents have a higher charge density and strong interaction with the molecular sieve skeleton, which leads to strong structural guidance and stabilizes molecular sieve crystals [27]. Combined with the XRD patterns (Figure 5), the growth of the (111) crystal plane was promoted because the alkyl chain changed from ethyl to butyl, thereby causing a change in the morphology of the SAPO-34 molecular sieve. When the cation content of the ionic liquid was increased to 1-ethyl-2,3-dimethyl imidazolium or 1-butyl2,3-dimethyl imidazolium, the framework structure of the molecular sieve was changed, and the characteristic peaks of the AEL and AFI structures appeared.

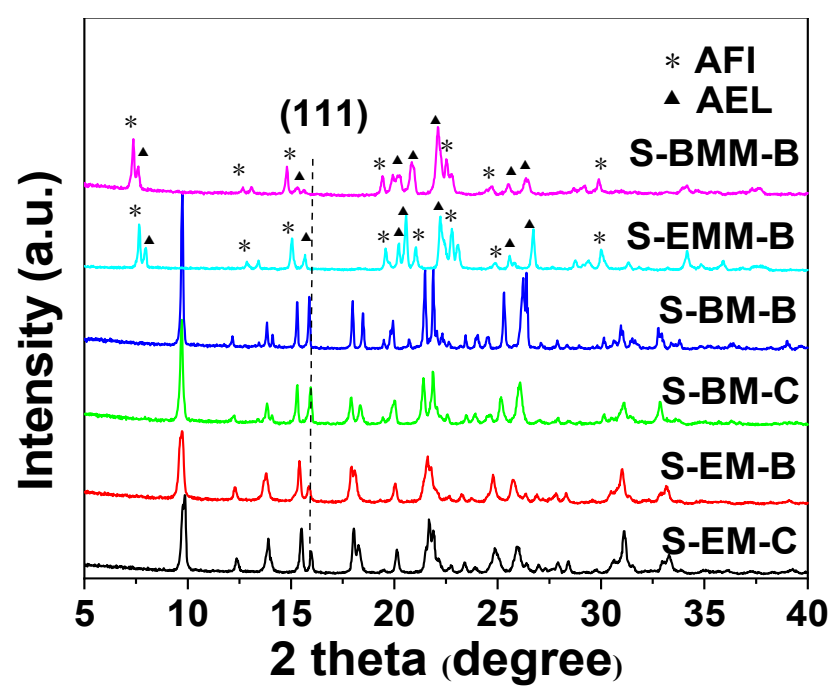

Figure 5. XRD patterns of samples synthesized with different ILs.

Since the study discussed above has shown that changing the cation can alter the morphology of the SAPO-34 molecular sieve, the next step was to investigate the effect of the anion. For this purpose, ionic liquids with the same cation but different anions were used. Hence, the SAPO-34 molecular sieves synthesized in either [EMIm] Cl (S-EM-C) or [BMIm] Cl (S-BM-C) were compared to those obtained in either [EMIm]Br (S-EM-B) or [BMIm]Br (S-BM-B). The overall morphology of S-EM-C and S-EM-B is spherical, and both S-BM-C and S-BM-B show cubic crystal morphology (Figure 6). However, because the anion of the ionic liquid determines its polarity and coordination ability, the coordination ability of $\mathrm{Cl}^{-}$is weaker than that of $\mathrm{Br}^{-}$[28]. Therefore, [EMIm]Cl and [BMIm]Cl systems provide an environment of weak coordination and strong polarity, and the reaction in which ionic liquid cations participate is accelerated. In [EMIm] Cl, the nucleation rate is accelerated, so sample S-EM-C is stacked by small cubes, and in the [BMIm]Cl system, the generation rate of aluminosilicate ions cannot meet the needs of crystal growth, so S-BM-C is scratched and the apex angle is defective while the surface of sample S-BM-B is smooth.

Therefore, ionic liquids have a great influence on the morphology and framework type of molecular sieves, so to a certain extent, ionic liquids can be used as SDAs. 


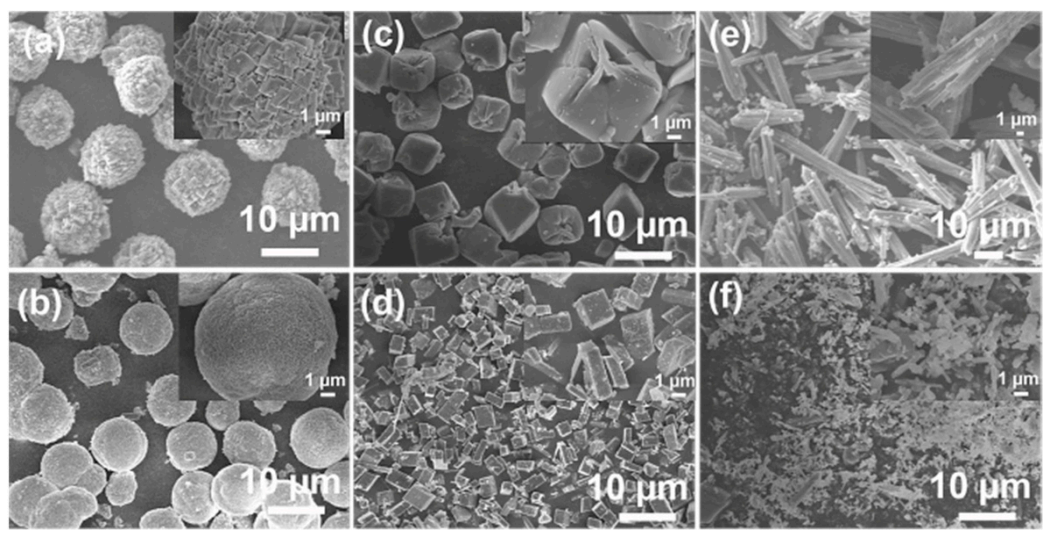

Figure 6. SEM images of samples synthesized with different ILs ((a) for S-EM-C, (b) for S-EM-B, (c) for S-BM-C, (d) for S-BM-B, (e) for S-EMM-B, (f) for S-BMM-B).

The solid-state ${ }^{13} \mathrm{C}$ nuclear magnetic resonance (NMR) spectra of sample S-EM-C were characterized to further study the role of [EMIm]Cl in the synthesis of triclinic SAPO-34 molecular sieves. As we can see in Figure 7, peaks at $132 \mathrm{ppm}$ and $125 \mathrm{ppm}$ belong to the carbon atoms on the imidazole ring of the ionic liquid. The two peaks of the N-C-C-N bond in [EMIm] Cl overlap into one peak (125 ppm) in the solid ${ }^{13} \mathrm{C}$ NMR spectrum. The $39 \mathrm{ppm}$ peak belongs to the substituted methyl group on the imidazole ring [23]. Peaks at $11 \mathrm{ppm}$ and $44 \mathrm{ppm}$ are attributed to substituted ethyl groups on the imidazole ring, which indicates that [EMIm] $\mathrm{Cl}$ exists in the framework structure of the molecular sieve.<smiles></smiles>

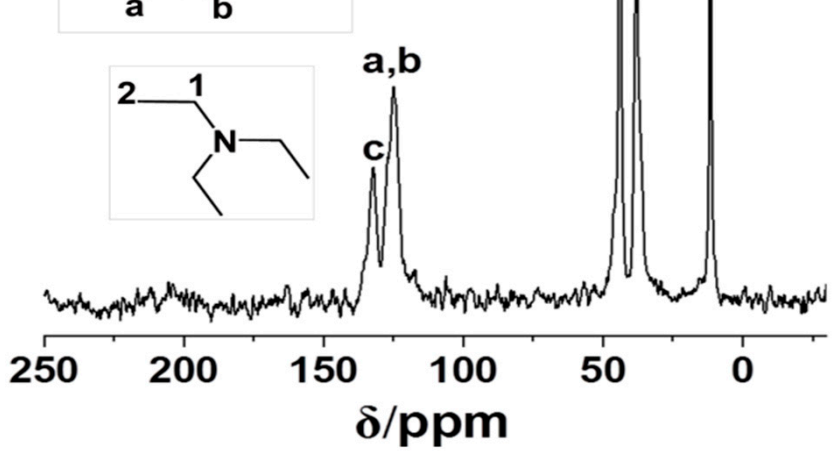

Figure 7. ${ }^{13} \mathrm{C}$ NMR spectra of sample S-EM-C.

\subsection{Effect of Organic Amines}

First, the influence of TEA dosage was investigated in [EMIm] $\mathrm{Cl}$ and [BMIm]Cl. The XRD patterns (Figure 8) show that [EMIm] Cl can be used as an SDA. With the addition of TEA, the relative crystallinity of the samples gradually increased. With increasing molar ratio of TEA to 1.5, a diffraction peak of the AEL molecular sieve appears. In [BMIm] Cl, when TEA is not added or the amount of TEA is too small, the samples (S2-T-0 and S2-T-0.5) show the characteristic peak of the aluminum phosphate dense phase, and there is no CHA topological structure characteristic peak. With increasing molar amount of TEA, the characteristic diffraction peaks of triclinic SAPO-34 molecular sieves with high crystallinity appeared in samples S2-T-1.0 and S2-T-1.5. The morphology of samples with different molar amounts of TEA is shown in Figures S9 and S10. 


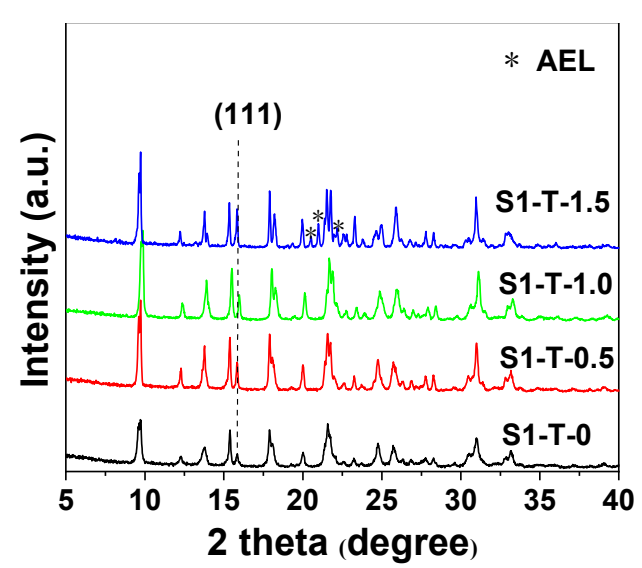

(a)

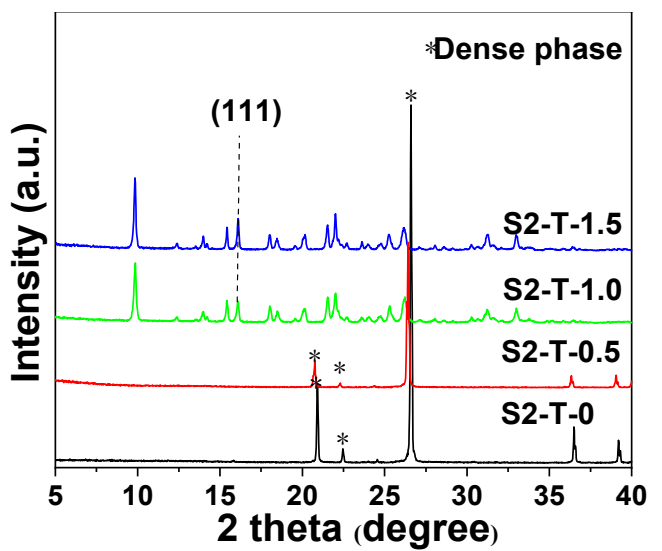

(b)

Figure 8. XRD patterns of samples under different molar amounts of TEA: (a) [EMIm]Cl and (b) $[\mathrm{BMIm}] \mathrm{Cl}$.

The amount of TEA has a significant influence on the synthesis of the SAPO-34 molecular sieve in [BMIm]Cl, so the influence of organic amines in [BMIm]Cl was investigated. Different kinds of organic amines (TEA, DEA, MOR, N-MIM, TEAOH) were added to explore the influence of organic amine types on the SAPO-34 molecular sieve. The structure of different organic amines is shown in Figure S11.

Figure S12 shows the XRD patterns of samples with different types of organic amines. Except for sample S2-TEAOH, the characteristic diffraction peaks of CHA topology appear at $2 \theta=9.68^{\circ}, 15.97^{\circ}, 20.2^{\circ}, 26.0^{\circ}$ and $31.0^{\circ}$. However, the characteristic peak of the dense phase of aluminum phosphate appears in S2-DEA. The peaks at $2 \theta=9.68^{\circ}$ and $15.97^{\circ}$ change in most of the samples synthesized by adding different organic amines, which proves the growth of the (100) and (111) crystal planes of the triclinic SAPO-34 molecular sieve. Figure S13 shows SEM images of samples with different types of organic amines. Due to the unsuitable molar amount of TEAOH in sample S2-TEAOH, the pH environment of the synthesis system (less than 4.6) is unsuitable for the formation of the triclinic SAPO34 molecular sieve, so the dense phase of aluminum phosphate is formed, showing an irregular sphere or cubic morphology. Sample S2-TEA shows a cubic morphology with a crystal size of approximately $6 \mu \mathrm{m}$, which exhibits nicks on the surface of the cubic crystal along the diagonal. Samples S2-DEA and S2-MOR are nanometer-scale cubic crystals. Sample S2-N-MIM is a scaly crystal with an average crystal size of $1 \mu \mathrm{m}$.

${ }^{13} \mathrm{C}$ NMR spectroscopy was performed to investigate the organic templates in the channels. Figure 9 shows the ${ }^{13} \mathrm{C}$ NMR spectra of samples with different types of templates. All samples have a strong peak at 37 ppm attributed to the methyl carbon; however, the characteristic peaks of four carbons on the butyl substituent of the ionic liquid do not exist. The peaks at $125 \mathrm{ppm}$ and $130 \mathrm{ppm}$ correspond to the carbon atoms on the imidazole ring [12]. Therefore, it can be clearly confirmed that the ionic liquid in the triclinic SAPO-34 molecular sieve is a 1,3-dimethylimidazole cation, which proves that [BMIm]Cl decomposes and reacts to generate 1,3- dimethylimidazole cation because of the addition of HF. In addition, the solid ${ }^{13} \mathrm{C}$ NMR spectra of all samples also show the resonance peaks of the organic amines [29], and the carbon atoms corresponding to the resonance peaks at different positions are shown in Figure 9.

The above results show that organic amines and ionic liquid cations fill the pores of the triclinic SAPO-34 molecular sieve together, forming a good mixed template system, which leads to the formation of products and inhibits the transition from a metastable molecular sieve structure to a thermodynamically stable inorganic dense phase. The template will significantly affect the particle size and physical and chemical properties of zeolite, and the addition of organic amine can adjust the crystal size, morphology and growth direction of the molecular sieve. 

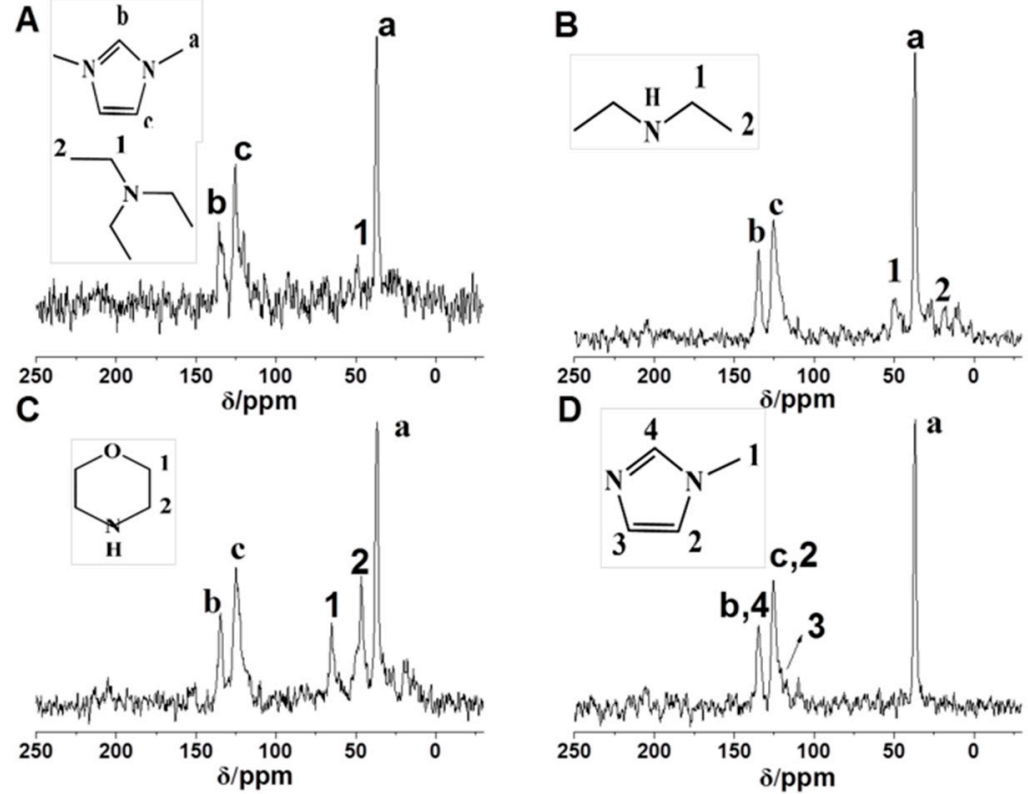

Figure 9. ${ }^{13} \mathrm{C}$ NMR spectra of samples under different types of templates ((A): S2-TEA, (B): S2-DEA, (C): S2-MOR, and (D): S2-N-MIM).

Figure 10 shows Thermogravimetric-Derivative thermogravimetry (TG-DTG) curves of triclinic SAPO-34 molecular sieves with (sample S1-T-1.0) and without TEA (sample S1T-0). Both S1-T-0 and S1-T-1.0 have two distinct stages: the removal of physically adsorbed water (near $100{ }^{\circ} \mathrm{C}$ ) and the removal of organic matter in the region of $250 \sim 500{ }^{\circ} \mathrm{C}$ [12]. It is obvious that the total weight loss rate of sample S1-T-1.0 (32.38\%) is greater than that of sample S1-T-0 (24.44\%). In the region of $200 \sim 500{ }^{\circ} \mathrm{C}$, the weight loss rate of sample S1-T-1.0 is $26.60 \%$, while the weight loss rate of sample S1-T-0 is $23.29 \%$, and the extra weight loss is attributed to the combustion of TEA [30].
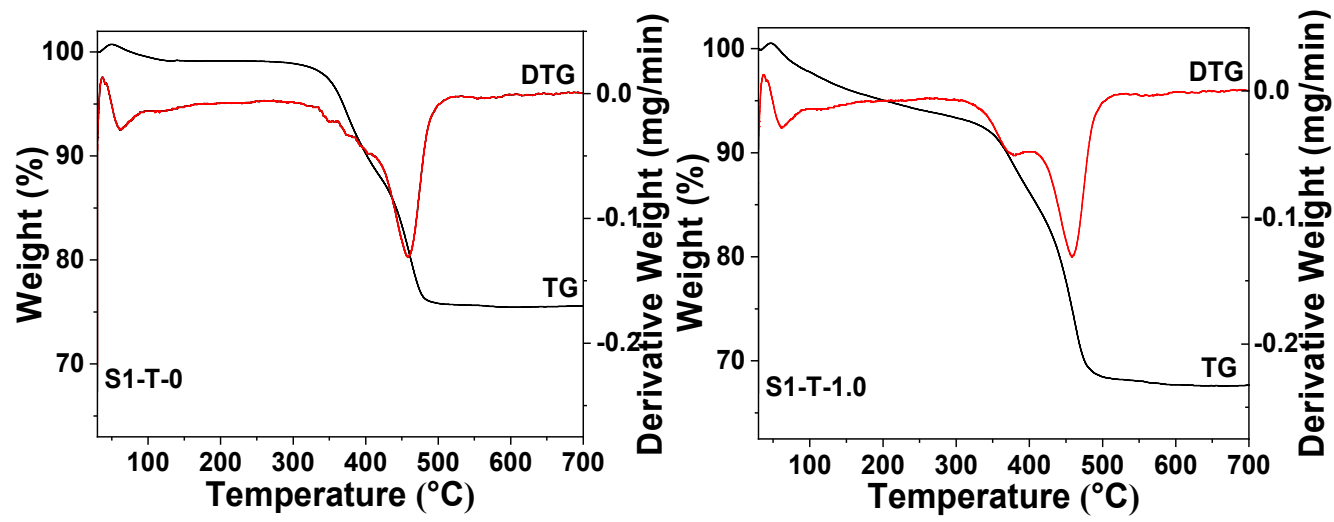

Figure 10. Thermogravimetric-Derivative thermogravimetry (TG-DTG) curves of samples under different TEA molar amounts.

Based on the above results, TEA and ionic liquids play a common structural guiding role in the synthesis of molecular sieves.

\subsection{Catalyst Test}

The product distribution and selectivity are related to the acidity of the catalysts in the methanol conversion reaction [31]. It can be seen from Figure S14 that there is a strong acid peak near $430{ }^{\circ} \mathrm{C}$ in S0, and the amount of strong acid in S0 is more than that of S1-Si-0.3. As shown in Table 1 , the dominant products are markedly different in the methanol conversion 
reaction over both S1-Si-0.3 and S0. The main products over S1-Si-0.3 and S0 are dimethyl ether (DME, 72.2\%) and light olefins (69\%), respectively. S1-Si-0.3, which was prepared by the ionothermal method, is suitable for high-selective DME production because of its low acidity [31].

Table 1. Results of MTO catalytic reaction.

\begin{tabular}{|c|c|c|c|c|c|c|c|c|c|c|c|c|}
\hline \multirow{2}{*}{ Sample } & \multirow{2}{*}{ Time } & \multirow{2}{*}{ Conversion/\% } & \multicolumn{10}{|c|}{ Yield $/ \%$} \\
\hline & & & $\mathrm{C}_{1}$ & $C_{2}=$ & $\mathrm{C}_{2}$ & $C_{3}=$ & $\mathrm{C}_{3}$ & DME & $\mathrm{C}_{4}=$ & $\mathrm{C}_{4}$ & $\mathrm{C}_{5}$ & $\mathrm{C}_{2}=\sim \mathrm{C}_{4}=$ \\
\hline So & $1 \mathrm{~h}$ & 100 & 26.7 & 44.7 & 0.5 & 21.0 & 0.6 & 0 & 3.3 & 1.7 & 1.5 & 69 \\
\hline S1-Si-0.3 & $1 \mathrm{~h}$ & 100 & 17.4 & 3.7 & 0.6 & 4.1 & 0.3 & 72.2 & 0.7 & 0.6 & 0.4 & 8.5 \\
\hline
\end{tabular}

\section{Materials and Methods}

\subsection{Materials}

Phosphoric acid $\left(\mathrm{H}_{3} \mathrm{PO}_{4}, 85\right.$ wt\% Sinopharm Chemical Reagent Co., Ltd., Beijing China), triethylamine (TEA, 99 wt\% Xilong Chemical Co., Ltd., Shanghai China), diethylamine (DEA, 99 wt\% Sinopharm Chemical Reagent Co., Ltd., Beijing China), tetraethyl ammonium hydroxide (TEAOH, $99 \mathrm{wt} \%$ Aladdin), N-methylimidazole (N-MIM, $99 \mathrm{wt} \%$ Beijing Huawei Ruike Technology Chemical Co., Ltd., Beijing China), morphine (MOR, $99 \mathrm{wt} \%$ Aladdin, Shanghai China), aluminum isopropoxide (98 wt\% Sigma-Aldrich, Shanghai Trade Co., Ltd., Shanghai China), hydrofluoric acid (HF, $40 \mathrm{wt} \%$ Luoyang Chemical Reagent Factory, Luoyang China), aqueous colloidal silica (28 wt\% Qingdao Ocean Factory, Qingdao China), [EMIm]Cl (AR), [EMIm]Br (AR), 1-butyl-3-methyl imidazolium chloride ([BMIm]Cl (AR)), 1-butyl-3-methyl imidazolium bromide ([BMIm]Br (AR)), 1-ethyl-2,3dimethyl imidazolium bromide ([EMMIm]Br (AR)) and 1-butyl-2,3-dimethylimidazolium bromide ([BMMIm]Br (AR)) were purchased from Lanzhou Institute of Physical Chemistry, Chinese Academy of Sciences.

\subsection{Catalyst Preparation}

Preparation of $\mathrm{AlPO}_{4}-34$. A total of $8.66 \mathrm{~g}$ of [EMIm]Cl ionic liquid was placed in a clean three-port flask, connected to a condenser tube, and then the three-port flask was placed in a $75^{\circ} \mathrm{C}$ water bath. The following materials were added under stirring: $0.52 \mathrm{~g}$ of $\mathrm{H}_{3} \mathrm{PO}_{4}, 48 \mu \mathrm{L}$ of $\mathrm{HF}, 0.32 \mathrm{~g}$ of isopropanol aluminum and $0.15 \mathrm{~g}$ of TEA. The synthesized gel (SG) was crystallized at $180^{\circ} \mathrm{C}$ for $48 \mathrm{~h}$. The obtained product was washed with deionized water until neutral, then placed in a $100{ }^{\circ} \mathrm{C}$ oven to dry to obtain a synthetic product denoted as $\mathrm{AlPO}_{4}-34$.

Preparation of SAPO-34 by hydrothermal method. Firstly, $2.07 \mathrm{~g}$ isopropanol aluminum and $12.86 \mathrm{~g}$ deionized $\mathrm{H}_{2} \mathrm{O}$ were stirred for $1 \mathrm{~h}$. Then, $5.18 \mathrm{~g} \mathrm{H}_{3} \mathrm{PO}_{4}$ was added to the above mixed solution and stirred for $2 \mathrm{~h}$. Next, $0.48 \mathrm{~g}$ silica sol was added and stirred for $1 \mathrm{~h}$. Then, $4.60 \mathrm{~g}$ TEA was added dropwise to the above mixture, stirred for $2 \mathrm{~h}$ to form a uniform synthetic gel, transferred to a reaction kettle with a polytetrafluoroethylene lining, and put into $180{ }^{\circ} \mathrm{C}$ oven for $48 \mathrm{~h}$. The product was washed and dried to obtain a sample, denoted S0.

Preparation of SAPO-34. First, a certain amount of ionic liquid was placed in a clean three-port flask, connected to a condenser tube, and then the three-port flask was placed in a $75{ }^{\circ} \mathrm{C}$ water bath. The following materials were added under stirring: $0.52 \mathrm{~g}$ of $\mathrm{H}_{3} \mathrm{PO}_{4}$, a certain amount of $\mathrm{HF}, 0.32 \mathrm{~g}$ of isopropanol aluminum, a certain amount of TEA and a certain amount of silica sol. The synthesized gel (SG) was crystallized at a certain temperature for certain hours. The obtained product was washed with deionized water until neutral, and then placed in a $100{ }^{\circ} \mathrm{C}$ oven to dry to obtain synthetic product.

In the [EMIm] $\mathrm{Cl}$ ionic liquid, samples synthesized at different times were denoted S1- $t$ $\mathrm{h}$, where $t$ indicates the crystallization time. Samples synthesized at different temperatures were denoted S1 $\left(T^{\circ} \mathrm{C}\right)$, where $T$ indicates the crystallization temperature. Samples synthesized with different amounts of silicon were denoted S1-Si- $x$, where $x$ indicates the molar 
ratio of $\mathrm{Si}$, and samples synthesized with different TEA amounts were denoted S1-T- $y$, where $y$ represents the molar ratio of TEA. Samples synthesized with different HF amounts were denoted S1-F- $z$, where $z$ represents the molar ratio of HF.

In the $[\mathrm{BMIm}] \mathrm{Cl}$ ionic liquid, samples synthesized with different TEA amounts were denoted S2-T-y, where y represents the molar ratio of TEA, and samples synthesized in different kinds of templates were denoted S2-TEA, S2-DEA, S2-MOR, S2- N-MIM, and S2-TEAOH.

Samples synthesized in different ionic liquids ([EMIm]Cl, [EMIm]Br, [BMIm]Cl, [BMIm] Br, [EMMIm]Br and [BMMIm]Br) were denoted S-EM-C, S-EM-B, S-BM-C, S-BM-B, S-EMM-B and S-BMM-B.

The specific synthesis conditions of all samples are shown in Tables S3-S5.

\subsection{Characterization}

Powder X-ray diffraction (XRD) patterns were obtained on a D8 ADVANCE X-ray diffractometer. The crystal size and structural characteristics were observed by scanning electron microscope (SEM, JSM-7500F) equipped with energy dispersive spectrometry (EDS). The surface element content of the sample was determined by EDS. The functional group structure of the sample was determined on a Nicolet iS50 Fourier infrared spectrometer (FT-IR) from Thermo Company in the United States. Nitrogen adsorption/desorption experiments were performed on a Micromeritics ASAP-2460 automatic adsorption instrument. The zeolites were all outgassed at $150{ }^{\circ} \mathrm{C}$ for $6 \mathrm{~h}$ before measurement. An American PerkinElmer STA 8000 synchronous analyzer was used for thermogravimetric analysis of the samples, and the temperature was increased from $25^{\circ} \mathrm{C}$ to $800{ }^{\circ} \mathrm{C}$ at a rate of $10^{\circ} \mathrm{C} / \mathrm{min}$ in an argon atmosphere. ${ }^{13} \mathrm{C}$ NMR and ${ }^{29} \mathrm{Si}$ NMR measurements were performed by a Swiss AVANCE (3) 400 WB nuclear magnetic resonance instrument.

Temperature-programmed desorption of ammonia $\left(\mathrm{NH}_{3}-\mathrm{TPD}\right)$ was performed on a multifunction adsorption instrument. The $\mathrm{NH}_{3}$-TPD process was determined using $\mathrm{N}_{2}$ as the carrier gas with a flow rate of $30 \mathrm{~mL} \cdot \mathrm{min}^{-1}$, and the activation and desorption temperature were $500{ }^{\circ} \mathrm{C}$ and $100-600{ }^{\circ} \mathrm{C}$.

The methanol conversion reaction was tested on a fixed-bed reactor. The amount of calcined catalyst (40-60 mesh) was $0.3 \mathrm{~g}$ and the reaction temperature was $450{ }^{\circ} \mathrm{C}$. The $\mathrm{N}_{2}$ flow rate was $30 \mathrm{~mL} \cdot \mathrm{min}^{-1}$, and the mass space velocity (WHSV) was $3 \mathrm{~h}^{-1}$. On-line analysis of product components was performed using a hydrogen ion flame detector (FID) and a Plot-Q column. Methanol conversion and product selectivity were calculated by the area correction normalization method.

\section{Conclusions}

Triclinic SAPO-34 molecular sieves were prepared using ionothermal synthesis. The morphology and crystallinity of the SAPO-34 molecular sieve can be controlled by the crystallization temperature, organic amines and the type of ionic liquids. A lower crystallization temperature is beneficial for the formation of flaky crystals, while a higher crystallization temperature is beneficial for the generation of large-size cubic crystals. The type of ionic liquid has a great influence on the synthesis of SAPO-34 molecular sieves. The morphology of the sample changed from spherical to cubic as the cation of the ionic liquid was changed from 1-ethyl-3-methyl imidazolium to 1-butyl-3-methyl imidazolium. Continuing to increase the size of the cation is beneficial for the formation of large pore size AEL and AFI molecular sieves. Different templates have different effects on the growth of the (100) and (111) crystal planes of SAPO-34 zeolite. The template agent with a smaller main structure contributes to the development of a smaller grain size. In addition, it can be proven through NMR and TG analyses that ionic liquids and organic amines can be used as SDAs together.

Supplementary Materials: The following are available online at https:/ /www.mdpi.com/article/10 .3390 / catal11050616/s1, Figure S1: XRD patterns of samples under different molar amounts of Si. Figure S2: SEM images of samples under different molar amounts of Si. Figure S3: XRD patterns of 
samples under different molar amounts of HF. Figure S4: SEM images of samples under different molar amounts of HF. Figure S5: (a) $\mathrm{N}_{2}$ adsorption-desorption isotherms and (b) BJH pore size distributions of triclinic SAPO-34 molecular sieves. Figure S6: XRD patterns of samples at different crystallization times. Figure S7: SEM images of samples at different crystallization times. Figure S8: Structure of ionic liquids. Figure S9: SEM images of samples under different molar amounts of TEA. Figure S10: SEM images of samples at different molar amounts of TEA. Figure S11: Structure of different templating agents. Figure S12: XRD patterns of samples under different types of template. Figure S13: SEM images of samples under different types of template. Figure S14: NH3-TPD patterns of SAPO-34 molecular sieves. Table S1: XRD date of different samples. Table S2: Structural parameters of SAPO-34 molecular sieves. Table S3: Synthesis conditions in [EMIm]Cl. Table S4: Synthesis conditions in different ionic liquids. Table S5: Synthesis conditions in different templates.

Author Contributions: Conceptualization, L.H.; experiment performed by X.Y.; methodology, X.Y., Y.D. and L.G.; validation, L.H. and J.W.; BET, FT-TR, SEM analysis, T.L., Y.Z., and J.X.; data curation, L.H.; writing-original draft preparation, L.H. and X.Y.; writing-review and editing, L.H., X.Y., and L.G.; supervision, L.H.; funding acquisition, L.H., T.L. and Z.W. All authors have read and agreed to the published version of the manuscript.

Funding: This research was funded by the NSFC-Henan Talent Development Joint Fund (Grant No. U1204215), National Natural Science Foundation of China (Grant No. 21802125), Foundation of State Key Laboratory of High-efficiency Utilization of Coal and Green Chemical Engineering (Grant No. 2020-KF-23).

Acknowledgments: We very grateful to the teachers of the School of Chemical Engineering for their support in the inspection equipment used for experiments.

Conflicts of Interest: The authors declare that they have no conflict of interest.

\section{References}

1. Davis, M.E. Ordered porous materials for emerging applications. Nature 2002, 417, 813-821. [CrossRef] [PubMed]

2. Ackley, M.W.; Rege, S.U.; Saxena, H. Application of natural zeolites in the purification and separation of gases. Microporous Mesoporous Mater. 2003, 61, 25-42. [CrossRef]

3. Davis, M.E. Zeolites from a Materials Chemistry Perspective. Chem. Mater. 2013, 26, 239-245. [CrossRef]

4. Sun, Q.; Wang, N.; Guo, G.Q.; Chen, X.X.; Yu, J. Synthesis of tri-level hierarchical SAPO-34 zeolite with intracrystalline micromeso-macroporosity showing superior MTO performance. J. Mater. Chem. A 2015, 3, 19783-19789. [CrossRef]

5. Xi, D.Y.; Sun, Q.M.; Xu, J.; Cho, M.; Cho, H.S.; Asahina, S.; Li, Y.; Deng, F.; Terasaki, O.; Yu, J. In situ growth-etching approach to the preparation of hierarchically macroporous zeolites with high MTO catalytic activity and selectivity. J. Mater. Chem. A. 2014, 2, 17994-18004. [CrossRef]

6. Kim, S.; Liu, Y.J.; Moore, J.S.; Dixit, R.S.; Pendergast, J.G.; Sholl, D.; Jones, C.W.; Nair, S. Thin Hydrogen-Selective SAPO-34 Zeolite Membranes for Enhanced Conversion and Selectivity in Propane Dehydrogenation Membrane Reactors. Chem. Mater. 2016, 28, 4397-4402. [CrossRef]

7. Li, M.; Wang, Y.H.; Bai, L.; Chang, N.; Nan, G.Z.; Hu, D.; Zhang, Y.F.; Wei, W. Solvent-free synthesis of SAPO-34 nanocrystals with reduced template consumption for methanol-to-olefins process. Appl. Catal. A Gen. 2017, 531, 203-211. [CrossRef]

8. Wu, P.F.; Yang, M.; Sun, L.; Zeng, S.; Xu, S.; Tian, P.; Liu, Z.M. Synthesis of nanosized SAPO-34 with the assistance of bifunctional amine and seeds. Chem. Commun. 2018, 54, 11160-11163. [CrossRef]

9. Liu, Y.; Wang, L.; Zhang, J.; Chen, L.; Xu, H. A layered mesoporous SAPO-34 prepared by using as-synthesized SBA-15 as silica source. Microporous Mesoporous Mater. 2011, 145, 150-156. [CrossRef]

10. Morris, R.E.; Weigel, S.J. The synthesis of molecular sieves from non-aqueous solvents. Chem. Soc. Rev. 1997, $26,309-317$. [CrossRef]

11. Cooper, E.R.; Andrews, C.D.; Wheatley, P.S.; Webb, P.B.; Wormald, P.; Morris, R.E. Ionic liquids and eutectic mixtures as solvent and template in synthesis of zeolite analogues. Nature 2004, 430, 1012-1016. [CrossRef] [PubMed]

12. Parnham, E.R.; Morris, R.E. 1-Alkyl-3-methyl Imidazolium Bromide Ionic Liquids in the Ionothermal Synthesis of Aluminium Phosphate Molecular Sieves. Chem. Mater. 2006, 18, 4882-4887. [CrossRef]

13. Taubert, A.; Li, Z. Inorganic materials from ionic liquids. Dalton Trans. 2007, 7, 723-727. [CrossRef] [PubMed]

14. Seddon, K. Ionic liquids A taste of the future. Nat. Mater. 2003, 2, 363-365. [CrossRef]

15. Greaves, T.L.; Drummond, C.J. Solvent nanostructure, the solvophobic effect and amphiphile self-assembly in ionic liquids. Chem. Soc. Rev. 2013, 42, 1096-1120. [CrossRef] [PubMed]

16. Morris, R.E. Ionothermal synthesis-ionic liquids as functional solvents in the preparation of crystalline materials. Chem. Commun. 2009, 21, 2990-2998. [CrossRef] 
17. Ngo, H.L.; Lecompte, K.; Hargens, L.; Mcewen, A.B. Thermal properties of imidazolium ionic liquids. Thermochim. Acta 2000, 357, 97-102. [CrossRef]

18. Fortas, W.; Djelad, A.; Hasnaoui, M.A.; Sassi, M.; Bengueddach, A. Adsorption of gentian violet dyes in aqueous solution on microporous AlPOs molecular sieves synthesized by ionothermal method. Mater. Res. Express 2018, 5, 25018. [CrossRef]

19. Li, X.; Li, K.; Ma, H.; Xu, R.; Tao, S.; Tian, Z. Ionothermal synthesis of a CHA-type aluminophosphate molecular sieve membrane and its formation mechanism. Microporous Mesoporous Mater. 2015, 217, 54-62. [CrossRef]

20. Lee, J.H.; Kim, E.J.; López-Arbeloa, F.; Hong, S.B.; Camblor, M.A. Microporous aluminophosphates synthesized with 1,2,3trimethylimidazolium and fluoride. Dalton. Trans. 2016, 45, 7616-7626. [CrossRef]

21. Lin, Y.; Wei, Y.; Zhang, L.; Guo, K.; Wang, M.; Huang, P.; Meng, X.; Zhang, R. Facile ionothermal synthesis of SAPO-LTA zeotypes with high structural stability and their catalytic performance in MTO reaction. Microporous Mesoporous Mater. $2019,28,109611$. [CrossRef]

22. Zhao, X.; Wen, J.; Zhao, J.; Li, A.; Li, G.; Wang, X. Hierarchically structured SAPO-5 molecular sieve catalysts with tailored mesoporosity for alkylation reaction. J. Porous Mater. 2015, 22, 577-584. [CrossRef]

23. Sánchez-Sánchez, M.; Romero, Á.A.; Pinilla-Herrero, I.; Sastre, E. Ionothermal preparation of triclinic SAPO-34 and its catalytic performance in the MTO process. Catal. Today 2017, 296, 239-246. [CrossRef]

24. Azim, M.M.; Stark, A. The First Systematic Study on the Conditions Affecting the Ionothermal Synthesis of Silicoaluminophosphates. ChemistrySelect 2018, 3, 12495-12503. [CrossRef]

25. Tan, J.; Liu, Z.; Bao, X.; Liu, X.; Han, X.; He, C.; Zhai, R. Crystallization and Si incorporation mechanisms of SAPO-34. Microporous Mesoporous Mater. 2002, 53, 97-108. [CrossRef]

26. Blasco, T.; Chica, A.; Corma, A.; Murphy, W.J.; Agúndez-Rodríguez, J.; Pérez-Pariente, J. Changing the Si distribution in SAPO-11 by synthesis with surfactants improves the hydroisomerization/dewaxing properties. J. Catal. 2006, 242, 153-161. [CrossRef]

27. Wang, Y.S.; Peng, X.Y.; Tian, Z.J.; Lin, L.W. Research Progress in Ionothermal Synthesis of Molecular Sieves. Chin. J. Catal. 2012, 33, 39-50. [CrossRef]

28. Tian, Z.J.; Liu, H. Ionothermal synthesis of zeolitic molecular sieves: Properties, progress and prospect. Chem. Ind. Eng. Prog. 2015, 34, 1501-1510.

29. Pei, R.; Tian, Z.; Wei, Y.; Li, K.; Xu, Y.; Wang, L.; Ma, H. Ionothermal synthesis of AlPO4-34 molecular sieves using heterocyclic aromatic amine as the structure directing agent. Mater. Lett. 2010, 64, 2384-2387. [CrossRef]

30. Wang, C.; Yang, M.; Tian, P.; Xu, S.; Yang, Y.; Wang, D.; Yuan, Y.; Liu, Z. Dual template-directed synthesis of SAPO-34 nanosheet assemblies with improved stability in the methanol to olefins reaction. J. Mater. Chem. A 2015, 3, 5608-5616. [CrossRef]

31. Feng, J.; Guo, L.L.; Wang, Z.; Wang, B.S.; Wang, J.F.; Lu, T.L.; Xu, J.; Zhan, Y.Z.; Rawal, A.; Zhao, C.; et al. Effect of ionothermal synthesis on the acidity and catalytic performance of a SAPO-5 molecular sieve. ChemistrySelect 2019, 35, 10520-10524. [CrossRef] 